\title{
Quantitative Prediction of Sensitization in Austenitic Stainless Steel Accounting for Multicomponent Thermodynamic and Mass Balance Effects
}

\author{
Satish KOLLI, ${ }^{11 *}$ Thomas OHLIGSCHLÄGER ${ }^{2)}$ and David PORTER ${ }^{1)}$ \\ 1) Department of Materials and Production Technology, University of Oulu, Oulu, 90014 Finland. \\ 2) Tornio R\&D Center, Outokumpu, Tornio, 95490 Finland.
}

(Received on October 24, 2018; accepted on February 6, 2019)

\begin{abstract}
Double Loop Electrochemical Potentiokinetic Reactivation testing has been employed to experimentally determine the degree of sensitization (DOS) of an austenitic stainless steel subjected to isothermal heat treatment for various times in the temperature range $700-820^{\circ} \mathrm{C}$. For the different heat treatment conditions, the chromium concentration profiles across grain boundaries were calculated using the diffusion module in Thermo-Calc ${ }^{\circledR}$ based on the assumptions that sensitization is caused by grain boundary $\mathrm{M}_{23} \mathrm{C}_{6}$ precipitates and that local multicomponent equilibrium and flux balance exist at the carbide - matrix interface. Comparison of the experimental DOS values and the details of the chromium concentration profiles was used to establish a quantitative depletion factor that predicts sensitization at short annealing times.
\end{abstract}

KEY WORDS: sensitization; Thermo-Calc; precipitation; diffusion.

\section{Introduction}

Austenitic stainless steels possess wide applications in industry owing to their superior corrosion resistance and mechanical properties. However, exposure to temperatures around $500-900^{\circ} \mathrm{C}$ reduces the corrosion resistance of the material making it vulnerable to intergranular corrosion (IGC), i.e. sensitization. ${ }^{1)}$ Despite many decades of research into the topic, there is still a lack of a quantitative tool that can predict the presence of sensitization on the basis of steel composition and heat treatment history.

Acid immersion tests have been used for a long time to measure sensitization. These simply involve subjecting the sample under examination to a test medium, which attacks the Cr-depleted zones. Corrosion rates are evaluated qualitatively by microscopic observations or weight loss. ${ }^{2)}$ These are used as industrial acceptance tests and are standardized in ASTM A262-15. Electrochemical potentiokinetic reactivation (EPR), can also be used to measure the degree of sensitization. either using single loop (SL-EPR) or double loop (DL-EPR) tests. These tests are non-destructive, rapid and provide a quantitative measure of sensitization. In the SLEPR method, a polished sample is polarized for 2 minutes at $200 \mathrm{mV}$ vs SCE. The potential is then decreased at a rate of $6 \mathrm{~V} / \mathrm{hr}$ to the corrosion potential. This causes the reactivation of the specimen by the breakdown of the passive film covering the chromium depleted regions. The drawbacks of the SL-EPR method are that it requires measurement of the specimen grain size and the reactivation behaviour is

\footnotetext{
* Corresponding author: E-mail: satish.kolli@oulu.fi

DOI: https://doi.org/10.2355/isijinternational.ISIJINT-2018-715
}

sensitive to surface finish. ${ }^{1)}$ This led to the development of DL-EPR test method. In this method, the sample is polarized anodically through the active region into the passive region, causing the formation of passive layer. The second loop involves the scanning in reverse direction, by decreasing the potential to corrosion potential thus causing the breakdown of passive film on the chromium depleted regions. The ratio of reactivation current density $\left(\mathrm{I}_{\mathrm{r}}\right)$ to the activation current density $\left(\mathrm{I}_{\mathrm{a}}\right.$ ) gives the degree of sensitization (DOS). The normalization of DOS with grain size is not necessary and a 600-grit surface finish provides reliable data. ${ }^{3)}$

It has been suggested that sensitization is due to the precipitation of chromium-rich grain boundary carbides that have a detrimental effect either through chromium depletion, local stresses, soluble segregate formation, or microgalvanic effects. ${ }^{4-6)}$ However, the initial depletion of chromium adjacent to grain boundaries onto which $(\mathrm{CrFe})_{23} \mathrm{C}_{6}$ carbides (also known as $\mathrm{M}_{23} \mathrm{C}_{6}$ ) have precipitated is now widely accepted as the primary cause of sensitization. ${ }^{7-9)}$

Clearly, therefore, sensitization needs to be studied in terms of the characteristics of the chromium depleted zones. ${ }^{10)}$ They are 1) the depth of the chromium depletion, and 2) the width of the depleted zones. ${ }^{10-12)}$

Regarding the depth and width of the chromium depleted zones, Stawstrom and Hillert ${ }^{13)}$ developed a simple model for grain boundary precipitation in $18 \mathrm{Cr}-8 \mathrm{Ni}-\mathrm{C}$ (wt.\%) stainless steel, but this neglected multicomponent effects, i.e. the effect of nickel, both in the thermodynamic and kinetic modelling. Hall and Briant developed a simple thermodynamic and kinetic model to predict chromium concentration profiles. ${ }^{7)}$ The effect of multicomponent system was neglected by considering only chromium in the 
thermodynamic calculations. Was and Kruge ${ }^{14)}$ realized that to accurately model the thermodynamics of carbide precipitation, considering only binary interaction parameters was not sufficient enough and introduced a ternary interaction parameter in the free energy expression for the $\mathrm{Ni}-\mathrm{Cr}-\mathrm{Fe}-\mathrm{C}$ system to model quaternary $\mathrm{Ni}-\mathrm{Cr}-\mathrm{Fe}-\mathrm{C}$ system. They developed an integrated thermodynamic and kinetic model to predict chromium concentration profiles. However, multicomponent effects were not completely taken into account by considering approximate single ternary interaction term for a quarternary system. Bruemmer ${ }^{12)}$ developed a theoretically based empirically modified model for the quantitative prediction of degree of sensitization. He correlated chromium depletion characteristics directly to DOS as measured using DL-EPR tests. However, the chromium concentration in the austenite at the interface with the carbide was calculated based on reaction theory, which does not guarantee the flux balance of solute atoms at the chromium carbides. Also, interface chromium concentrations were not measured, but rather the concentrations on the grain boundaries between the carbides. Sahlaoui ${ }^{15)}$ developed a two-stage diffusional model taking into account dechromization and rechromization. The use of two stage description violates the assumption that the carbide-matrix interface should remain in local equilibrium during diffusion-controlled growth. Also, inaccuracy in predictions of $\mathrm{Cr}$ concentration profiles was observed due to the assumption that precipitation has finished by the time self-healing begins. Sourmail et al. ${ }^{16)}$ modelled grain boundary precipitation of carbides considering multicomponent effects with the use of MTDATA software for thermodynamic calculations. The interfacial $\mathrm{Cr}$ concentration was calculated by solving flux balance equations at the carbide-matrix interface. Nevertheless, no correlation between the calculated depletion characteristics and DOS was made.

It is clear, therefore, that a study relating experimentally measured degrees of sensitization to grain boundary chromium concentration profiles predicted on the basis of multicomponent thermodynamic and diffusion effects has yet to be published. Using such an approach, this paper demonstrates that DOS can be quantitatively related to heat treatment parameters through a chromium depletion factor that considers both the depth and width of predicted chromium grain boundary depletion profiles. Such an approach is required if we are to quantitatively predict the presence or absence of sensitization after industrial processing.

\section{Experiments and Methodology}

\subsection{Materials}

The investigated material was a $3 \mathrm{~mm}$ thick commercial

Table 1. Heat treatment parameters used in the Gleeble $3800^{\circledR}$ thermo-mechanical simulator.

\begin{tabular}{ccc}
\hline $\begin{array}{c}\text { Heating rate to peak } \\
\text { temperature }\left({ }^{\circ} \mathrm{C} / \mathrm{s}\right)\end{array}$ & $\begin{array}{r}\text { Peak temperature } \\
\left(\mathrm{PT},{ }^{\circ} \mathrm{C}\right)\end{array}$ & $\begin{array}{c}\text { Holding time } \\
(\mathrm{HT}, \text { mins })\end{array}$ \\
\hline 50 & 700 & $1,10,20,30,40,80$ \\
50 & 760 & $1,5,10,20,30,40$ \\
50 & 820 & $1,5,10,20,30,40$ \\
\hline
\end{tabular}

austenitic stainless steel of type EN1.4310 obtained from Outokumpu Stainless Oy, Tornio, Finland. The chemical composition of the steel in wt.\% was Fe-16.80Cr-6.36Ni$0.95 \mathrm{Si}-0.105 \mathrm{C}$. The material was first solution treated and homogenized at $1100^{\circ} \mathrm{C}$ for 2 hours followed by water quenching. ${ }^{11)}$ After this, the alloy was subjected to the heat treatments given in the Table 1 using a Gleeble $3800^{\circledR}$ thermomechanical simulator, i.e. heated to a peak temperature (PT) of 700,760 and $820^{\circ} \mathrm{C}$, held for different times (HT) and then cooled rapidly to room temperature.

\subsection{DL-EPR Test}

DOS values for the initial solution treated and for the heat treated samples were measured with the help of double loop electrochemical potentiokinetic reactivation testing according to the standard EN ISO 12732, ${ }^{3)}$ which also describes the limits of the DOS values defining highly sensitized, slightly sensitized and unsensitized states. Before the test, the samples were polished to a 600-grit finish. The DOS is not sensitive to surface finish as the forward, anodic polarization scan causes the dissolution of all the surface irregularities and establishes a passive film. ${ }^{17)}$ Polarization in the reverse direction causes the dissolution of this passive layer at any chromium depleted regions, which are susceptible to corrosion, and produces a reaction current peak. The ratio of reactivation current density to activation current density gives the DOS. The peak current density depends on the extent of Cr depletion. ${ }^{18)} 0.5 \mathrm{M} \mathrm{H}_{2} \mathrm{SO}_{4}+0.01 \mathrm{M} \mathrm{KSCN}$ was used as the medium for the test at ambient temperature with a scan rate of $6 \mathrm{~V} / \mathrm{h}$.

\section{Results}

The values of DOS obtained from the DL-EPR tests are plotted against time at the various peak temperatures for the investigated alloy in Fig. 1. All data points are means of two measurements which differed by at most $1 \%$ on the DOS scale. Therefore, error bars are not drawn. For all the holding temperatures, the magnitude of DOS increases with

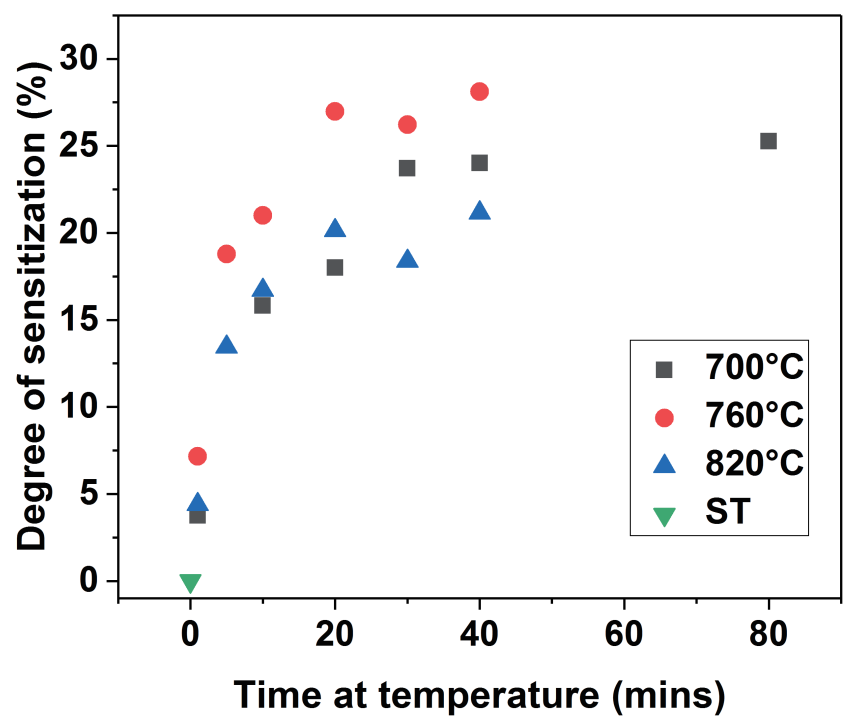

Fig. 1. \% Degree of sensitization values from DL-EPR tests for isothermal treatments (ST means solution treated and quenched, DOS =0). (Online version in color.) 
time at an ever decreasing rate, showing signs of saturation for the longest times. It can be seen that the DOS is highest for all times when holding at $760^{\circ} \mathrm{C}$. It seems, therefore that the precipitation of chromium carbides is faster at $760^{\circ} \mathrm{C}$ than at either 700 or $820^{\circ} \mathrm{C}$ implying that $760^{\circ} \mathrm{C}$ is closest to the temperature for the fastest precipitation kinetics, with $700^{\circ} \mathrm{C}$ having slower kinetics due to slower diffusion and $820^{\circ} \mathrm{C}$ a lower driving force. The precipitation kinetics are considered in detail below.

\section{Modelling}

Grain boundary precipitates will form at certain nucleation sites on the boundaries and can be expected to grow with the aid of both matrix and grain boundary diffusion into one or both grains as shown in Fig. 2. ${ }^{1,20)}$ Grain boundary diffusion of chromium should reduce the incubation time for precipitate nucleation on the boundary and in this work, it is assumed that the grain boundary nucleation of $\mathrm{M}_{23} \mathrm{C}_{6}$ starts immediately after reaching the isothermal temperatures so that the modelling problem can be reduced to one of carbide growth in an austenitic matrix. As will be shown, this approach seems to provide a satisfactory agreement with the DOS results. The mobility of the substitutional alloying elements along the grain boundaries is much higher than that through the austenite matrix ${ }^{21,22)}$ so that the grain boundaries can be expected to operate as collector plates for the substitutional atoms diffusing through the matrix to the grain boundaries. ${ }^{23}$ ) The high mobility along the grain boundaries also means that only low concentration gradients are required in the boundary plane to transport the substitutional atomic flux arriving from the matrix. It is reasonable to suppose, therefore, that the chromium

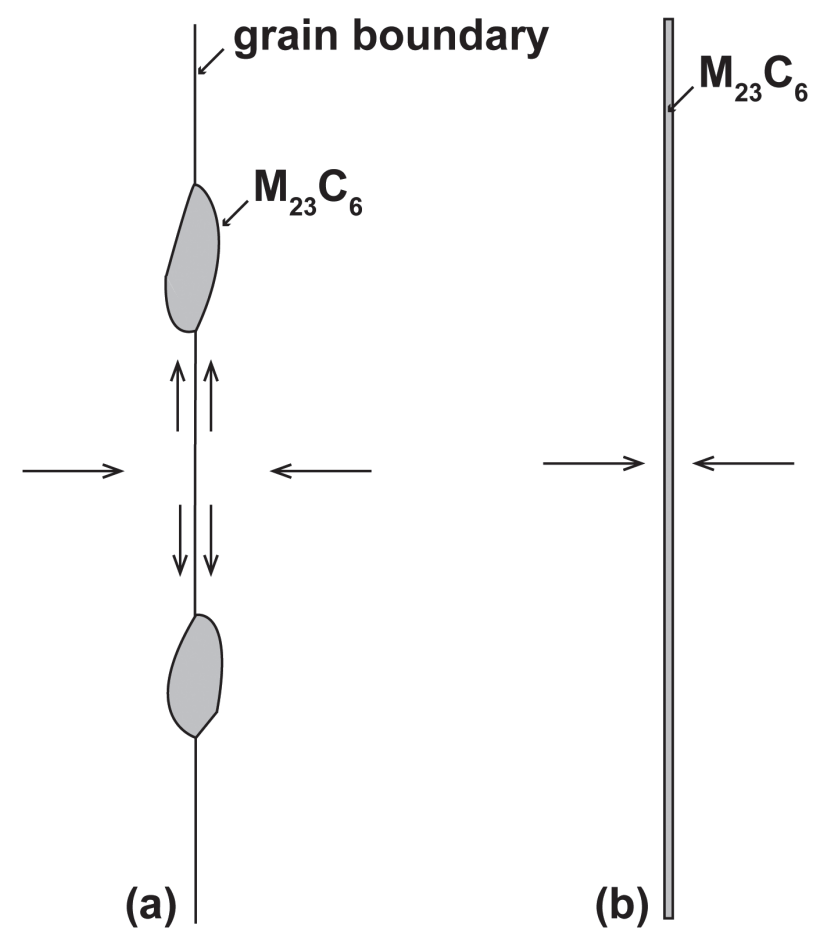

Fig. 2. (a) $\mathrm{M}_{23} \mathrm{C}_{6}$ precipitates on a grain boundary (g.b). The arrows show the diffusion flux of $\mathrm{Cr}$ towards and along the g.b. (b) the one dimensional representation of g.b precipitation assumed in the modelling. concentration profile perpendicular to a grain boundary will not vary much with position along the boundary. Assuming the precipitate growth is diffusion controlled rather than interface mobility controlled, the chromium concentration profile perpendicular to the grain boundary should be very similar to that which would exist ahead of an $\mathrm{M}_{23} \mathrm{C}_{6}$ precipitate film covering the boundary and advancing under diffusion control as shown in Fig. 2(b). Growth can then be modelled one dimensionally, which is the approach used here. ${ }^{15,16,24)}$ For the sake of the growth calculations, the initial half-thickness of the $\mathrm{M}_{23} \mathrm{C}_{6}$ film $d_{\mathrm{p}}$ is taken as $0.05 \mathrm{~nm}$. This consideration of $1 \mathrm{D}$ model (continuous precipitation) neglects the capillary effects as the curvature of individual nuclei and their radii are not considered in the calculations. Thus effecting the variation of interfacial concentrations and the solubility line during the growth of nuclei and resulting in the absence initial decrease in $\mathrm{Cr}_{\text {int }}$ during the growth of chromium carbides.

Growth of the grain boundary precipitates was modelled using the diffusion module in Thermo-Calc, i.e. TC_DICTRA, which is a tool for simulating diffusion in multicomponent alloys. The simulations assume local equilibrium at the austenite - carbide interface and are based on a numerical solution of multi-component diffusion equations combined with thermodynamic predictions of the carbide composition and the local equilibrium conditions at the carbide - austenite interface. ${ }^{25}$ ) The simulations were performed using the MOBFE2 mobility database and the TCFE7 thermodynamic database. Multi-component modelling was concerned, taking into account the elements $\mathrm{Fe}, \mathrm{Cr}$, $\mathrm{Ni}, \mathrm{Si}$ and $\mathrm{C}$ in the bulk concentrations given in section 2.1 .

A schematic isothermal section through the $\mathrm{Fe}-\mathrm{Cr}-\mathrm{C}$ ternary phase diagram can be seen in Fig. 3. The tie-line that governs the composition at the interface changes as growth proceeds. As $\mathrm{C}$ is an interstitial element and its diffusion coefficient is much higher than that of the other alloying elements, it was assumed that the activity of carbon is constant throughout the material. This iso-activity of

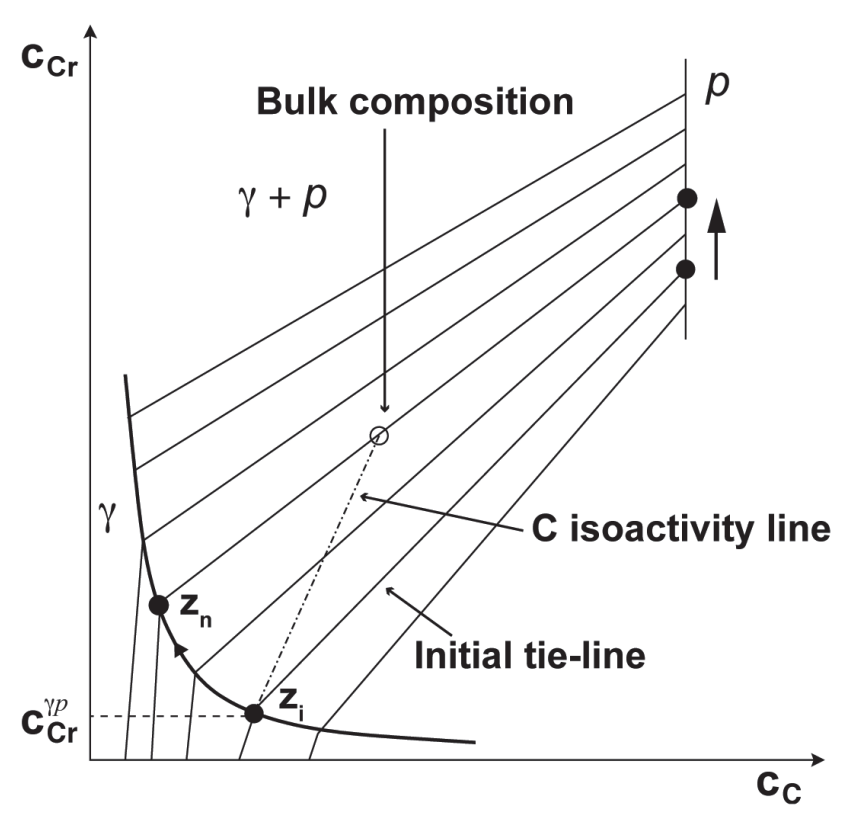

Fig. 3. Simple ternary phase diagram showing the change of equilibrium during precipitation. 
$\mathrm{C}$ is used to attain the conditions required for the assumed initial local equilibrium condition. The tie line that satisfies the bulk mass balance (final equilibrium) does not correspond to the isoactivity of $\mathrm{C}$. The initial composition at the interface is governed by the operative tie-line that is obtained by the iso-activity line for $\mathrm{C}$ that passes through the bulk composition of the material, which is given by $\mathrm{Z}_{\mathrm{i}}$ in Fig. 3. As precipitation proceeds, $\mathrm{C}$ activity decreases and the interfacial $\mathrm{Cr}$ concentration in the austenite increase $\left(\mathrm{z}_{\mathrm{i}}\right.$ to $\left.\mathrm{z}_{\mathrm{n}}\right)$ as indicated by the arrow. Figure 3 is a simplified $\mathrm{Fe}-\mathrm{Cr}-\mathrm{C}$ ternary representation of reality, which actually involves the elements $\mathrm{Fe}-\mathrm{Cr}-\mathrm{C}-\mathrm{Ni}-\mathrm{Si}$. In multicomponent systems, there are an infinite number of possible tie-lines governing the growth and finding the operating tie-line becomes difficult. To determine the composition on either side of the carbide - austenite interface in such cases, the following iterative method is used. Interfacial velocity $v^{\gamma}$

(where $p$ is carbide precipitate and $\gamma$ is austenite phase) and activity guesses are made and the conditions of local equilibrium at the interface are estimated to give the interfacial compositions $\left(c_{k}^{p}, c_{k}^{\gamma}\right)$ that can be used as boundary conditions. A check is then made as to whether the flux balances at the interface are satisfied, i.e. whether the rates of consumption of the various elements $k$ at the advancing interface are delivered to or removed from the interface by the diffusive flux. In other words, whether the following equation is satisfied:

$$
v^{\frac{p}{\gamma}}\left(c_{k}^{p}-c_{k}^{\gamma}\right)=J_{k}^{p}-J_{k}^{\gamma}
$$

where $J_{k}^{p}, J_{k}^{\gamma}$ are the fluxes of component $k$ at the carbide austenite interface during the current time step and $c_{k}^{\gamma}$ and

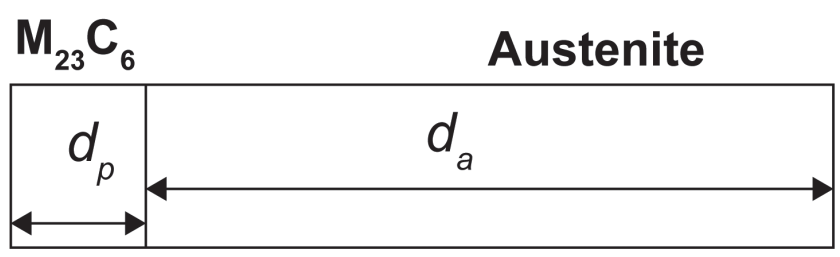

Fig. 4. Treatment of numerical simulation across the carbideaustenite interface. $c_{k}^{p}$ are the concentrations of element $k$ either side of the interface in the austenite and carbide phase respectively. If the flux balance equations are not satisfied, new interfacial velocities and activities are tried until the conditions are met and the operative tie-line is found. The procedure is then repeated for all time steps. ${ }^{26)}$

In defining the modelling conditions, it is necessary to take into account the soft-impingement of the precipitates on different grain boundaries, i.e. the overlap of the diffusion fields that occurs during precipitation. This was achieved in the present one-dimensional model in a simple way by choosing an appropriate austenite width from which $\mathrm{C}$ can be withdrawn, i.e. $d_{a}$ in Fig. 4. It seems appropriate, as suggested by Sourmail, ${ }^{27)}$ to assume $d_{a}$ is one sixth of the grain diameter. As soft-impingement is a three-dimensional problem, assuming a simple cubic grain shape, means that it is appropriate to attribute a sixth of the grain diameter to each one-dimensional calculation volume, as six faces draw $\mathrm{C}$ from the same grain. ${ }^{27)}$ In the present case, the mean linear intercept grain diameter was measured to be $72 \mu \mathrm{m}$ giving $d_{\mathrm{a}}=12 \mu \mathrm{m}$.

\section{Discussion of Modelling Results and Comparison with Experiments}

The calculated $\mathrm{Cr}$ concentration profiles across the carbide - austenite interface at the end of the isothermal treatments are of the form shown schematically in Fig. 5. $\mathrm{Cr}_{\text {int }}$ is the chromium concentration in the austenite at the interface. This value is a result of the local equilibrium condition assumed to exist at the mobile model interface. It depends on the isothermal holding temperature and changes with holding time as illustrated in Fig. 5. Following ${ }^{28)} \mathrm{Cr}_{\text {crit }}$ is a critical chromium concentration that determines an effective width $\left(\mathrm{W}_{\text {crit }}\right)$ of the chromium depleted zone at the grain boundary. The width $\mathrm{W}_{\text {crit }}$ initially increases with isothermal holding time and then decreases at longer times when the self-healing phase of sensitization becomes operative. ${ }^{28)}$ Sahlaoui ${ }^{29)}$ suggested that only $\mathrm{W}_{\text {crit }}$ is needs to be considered in to explain depletion characteristics and DOS. However, both $\mathrm{Cr}_{\text {int }}$ and $\mathrm{W}_{\text {crit }}$ are considered in characterising the $\mathrm{Cr}$ depletion and explaining DOS in this work. The concentration profiles of chromium, carbon, silicon and

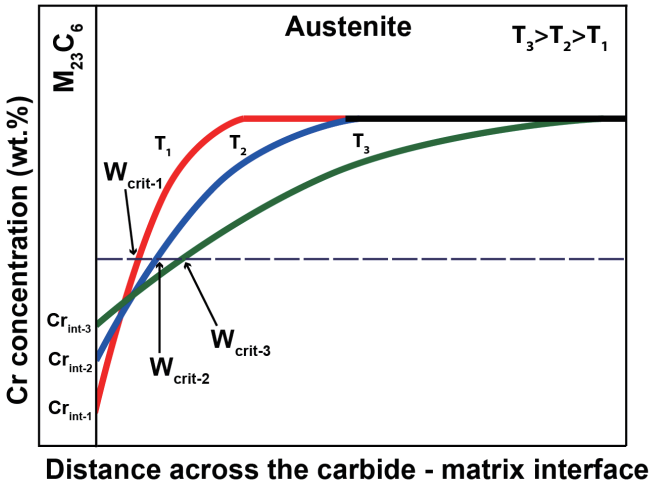

(a)

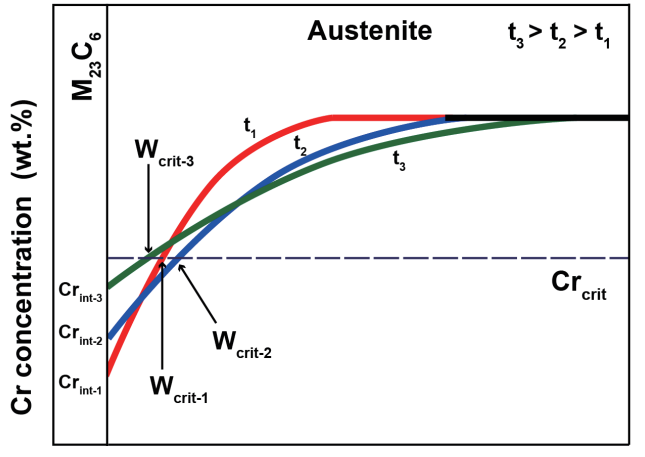

Distance across the carbide - matrix interface (b)

Fig. 5. Schematic calculated $\mathrm{Cr}$ concentration profiles in the austenite ahead of the $\mathrm{M}_{23} \mathrm{C}_{6}$ boundary film at the end of isothermal holding for a) different hold temperatures $T_{3}>T_{2}>T_{1}$, b) different holding times $t_{1}<t_{2}<t_{3}$. (Online version in color.) 
nickel obtained using DICTRA for the isothermal holding temperatures and times used in the experimental determination of DOS can be seen in Figs. 6-9.

In Fig. 6, a significant increase in $\mathrm{Cr}_{\text {int }}$, i.e. decreasing depth of the chromium depleted zone, with increasing temperature is predicted. $\mathrm{Cr}_{\text {int }}$ also increases with holding time, but only slightly over the time range involved. The width of the chromium depleted zone, $\mathrm{W}_{\text {crit }}$, depends, of course, on the choice of $\mathrm{Cr}_{\text {crit }}$. As discussed below, the most appropriate value of $\mathrm{Cr}_{\text {crit }}$ in the present study was found to be 12 wt.\% Cr, which is marked in Fig. 6. The figure shows that there is a significant increase in $\mathrm{W}_{\text {crit }}$ with increasing time, increasingly so as the temperature rises.

Carbon is depleted from the austenite over larger distances than chromium, as expected, Fig. 7. As shown in Fig. 8, silicon is rejected from the growing carbide into the austenite. However, a slight increase in silicon concentration in austenite is observed adjacent to the interface. Of course, the activity of $\mathrm{Si}$ decreases away from the interface, but the interactions of $\mathrm{Si}$ with $\mathrm{Ni}$ and $\mathrm{Cr}$ cause the $\mathrm{Si}$ concentration profile to take on the shape seen. As the solubility in the carbide of $\mathrm{Si}$ and $\mathrm{Ni}$ is minute, both $\mathrm{Si}$
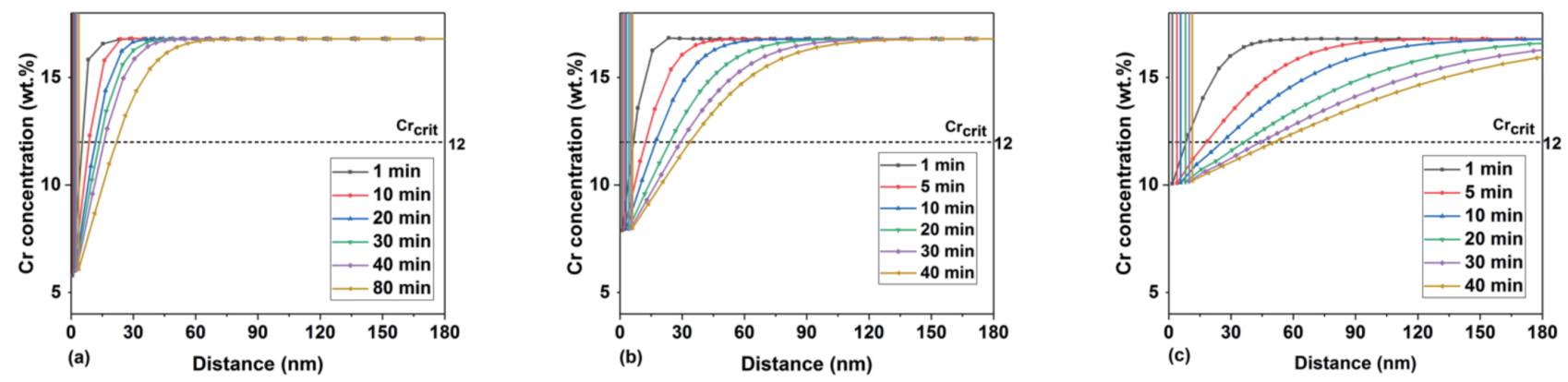

Fig. 6. $\mathrm{Cr}$ concentration profiles across $\mathrm{M}_{23} \mathrm{C}_{6}$ carbide - austenite matrix interface at (a) $700^{\circ} \mathrm{C}$ (b) $760^{\circ} \mathrm{C}$ (c) $820^{\circ} \mathrm{C}$. (Online version in color.)
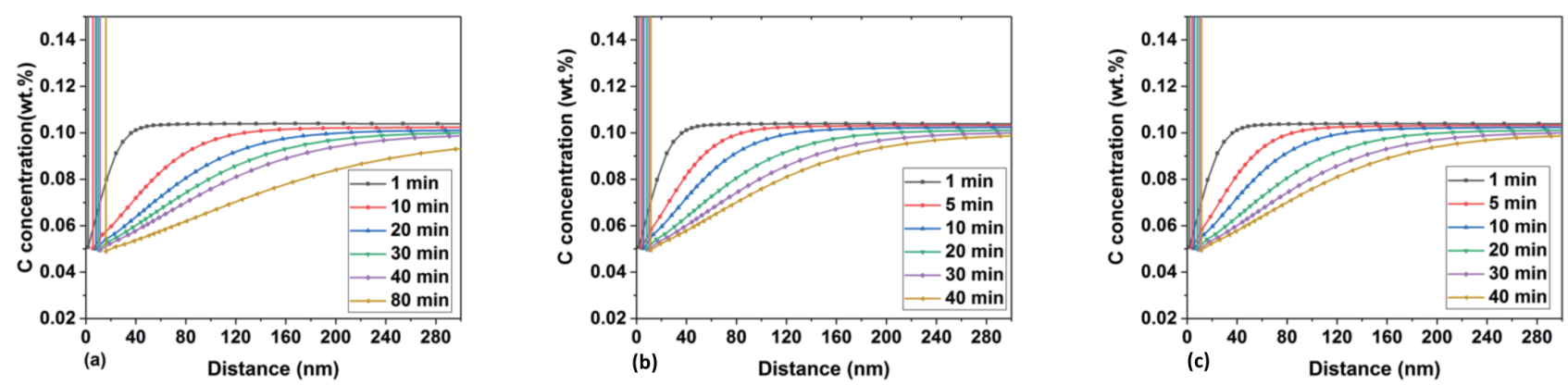

Fig. 7. $\mathrm{C}$ concentration profiles across $\mathrm{M}_{23} \mathrm{C}_{6}$ carbide - austenite matrix interface at (a) $700^{\circ} \mathrm{C}$ (b) $760^{\circ} \mathrm{C}$ (c) $820^{\circ} \mathrm{C}$. (Online version in color.)

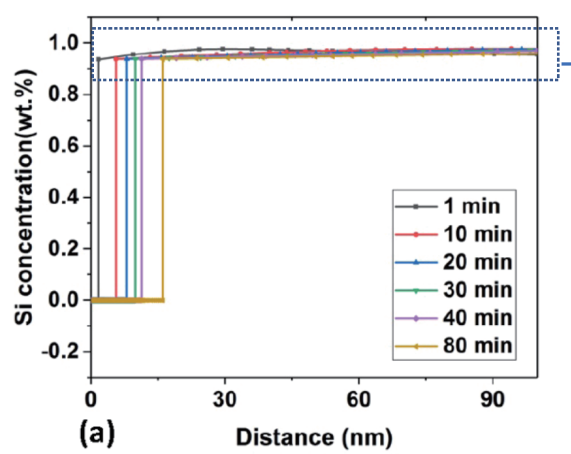

(a) Distance $(\mathrm{nm})$

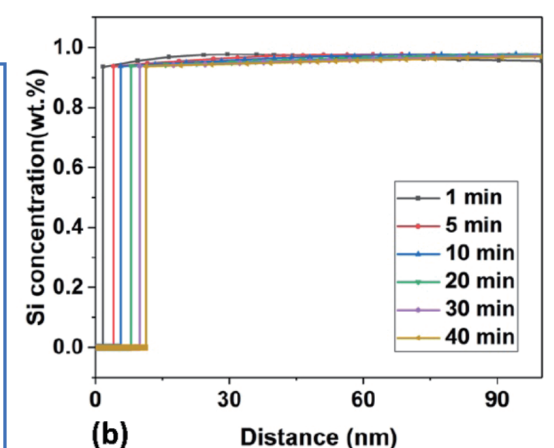

(b)

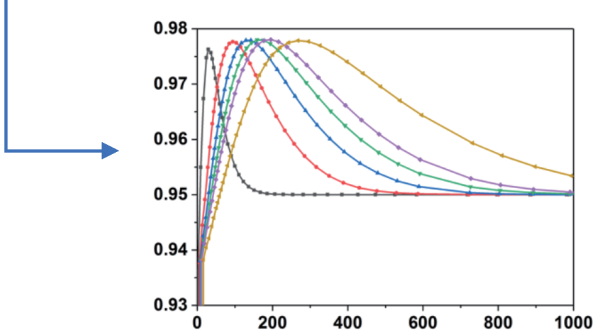

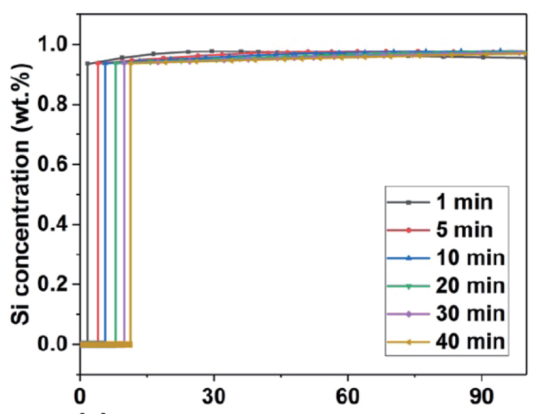

(c)

Fig. 8. Si concentration profiles across $\mathrm{M}_{23} \mathrm{C}_{6}$ carbide - austenite matrix interface at (a) $700^{\circ} \mathrm{C}$ (b) $760^{\circ} \mathrm{C}\left(\right.$ c) $820^{\circ} \mathrm{C}$. (Online version in color.) 

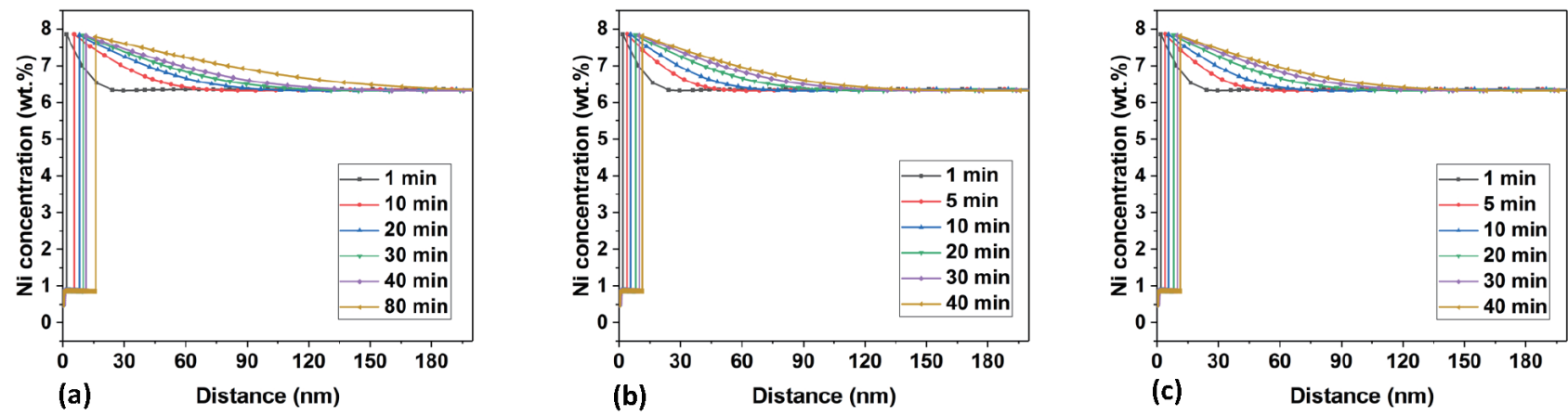

Fig. 9. Ni concentration profiles across $\mathrm{M}_{23} \mathrm{C}_{6}$ carbide - austenite matrix interface at (a) $700^{\circ} \mathrm{C}$ (b) $760^{\circ} \mathrm{C}$ (c) $820^{\circ} \mathrm{C}$. (Online version in color.)

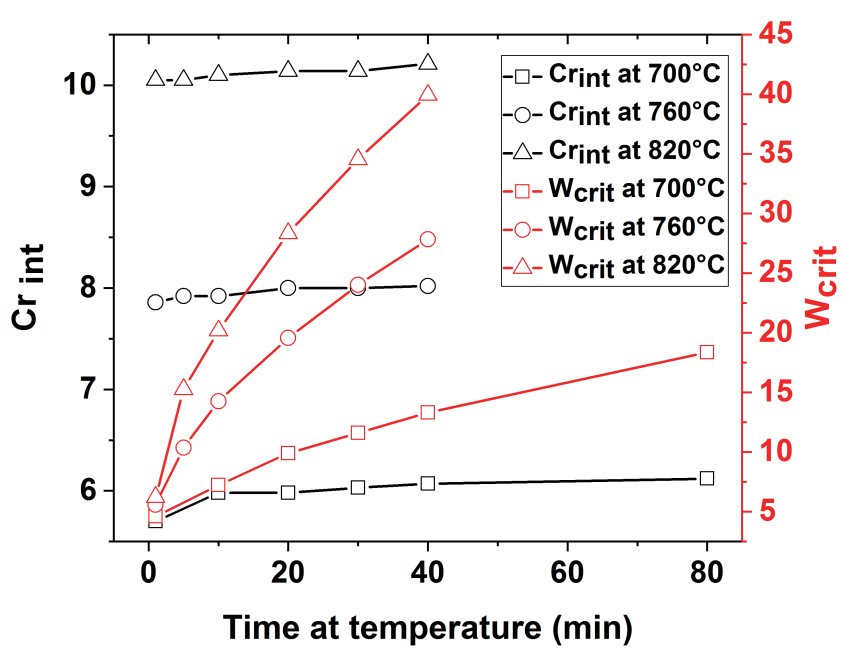

Fig. 10. Variation of $\mathrm{Cr}_{\text {int }}$ and $\mathrm{W}_{\text {crit }}$ at $12 \mathrm{wt} . \% \mathrm{Cr}$ with temperature and times at isothermal temperatures. (Online version in color.)

and $\mathrm{Ni}$ are rejected, while $\mathrm{Cr}$ is absorbed, from ahead of the advancing interface. $\mathrm{Cr}$ and $\mathrm{Ni}$ both reduce the activity of $\mathrm{Si}$, but the effect of $\mathrm{Ni}$ is responsible for the initial increase in $\mathrm{Si}$ concentration with increasing distance from the interface.

Returning to the chromium profiles in Fig. 6, the variation of $\mathrm{Cr}_{\text {int }}$ and $\mathrm{W}_{\text {rit }}$ with temperature and holding time can be seen in Fig. 10. Neither $\mathrm{Cr}_{\text {int }}$ or $\mathrm{W}_{\text {crit }}$ alone correlates with the experimentally measured DOS values in Fig. 1, as shown for example in Figs. 11(a), and 11(b). Following Bruemmer $^{3}$ it is reasonable to assume that the degree of sensitization will depend not only on the width of the chromium depleted zone as defined by some critical chromium concentration, but also on the depth of the depletion profile, which could be defined as $\mathrm{Cr}_{\text {int }}$ or $\left(\mathrm{Cr}_{\text {crit }}-\mathrm{Cr}_{\text {int }}\right)$, for example. It is possible to conceive of various depletion parameters that combine the terms $\mathrm{W}_{\text {crit }}, \mathrm{Cr}_{\text {crit }}$ and $\mathrm{Cr}_{\text {int }}$. Three such depletion parameters are $\left(\mathrm{Cr}_{\text {crit }}-\mathrm{Cr}_{\text {int }}\right) \cdot \mathrm{W}_{\text {crit }},\left(\mathrm{Cr}_{\text {crit }} / \mathrm{Cr}_{\text {int }}\right) \cdot \mathrm{W}_{\text {crit }}$ and $\mathrm{W}_{\text {crit }} / \mathrm{Cr}_{\text {int }}$. Statistical analyses of the correlations between the experimentally measured DOS values and these potential depletion parameters based on a variety of definitions of $\mathrm{Cr}_{\text {crit }}$ showed that the best correlation is obtained with the following depletion parameter:

$$
\mathrm{DP}=\left(12-\mathrm{Cr}_{\text {int }}\right) \cdot \mathrm{W}_{\text {crit }}
$$

i.e. using $\mathrm{W}_{\text {crit }}$ values defined by $\mathrm{Cr}_{\text {crit }}=12 \mathrm{wt} . \%$. The non-linear correlation between this parameter and the exper- imental DOS values is shown in Fig. 11(c). The correlation is clearly superior to that in Fig. 11(b), i.e. that based on $\mathrm{W}_{\text {crit }}$ alone. With the exception of one data point at the DP = $22.3 \mathrm{~nm} . \mathrm{wt} \%$, the DOS data points lie in a scatter band that is $+/-4$ DOS percentage points. The relationship between DOS and DP is seen to be non-linear, as might be expected, since very long heat treatment times eventually lead to selfhealing, i.e. a decrease in DOS.

It also seems that, for the present heat treatment conditions at least, the modelling of the precipitation process and development of $\mathrm{Cr}$ depleted zones can be reasonably done by assuming that precipitate growth starts immediately without any incubation time. The inclusion of an incubation time might reduce the scatter observed in the DOS - DP correlation, but would be more reasonable to consider for low temperature heat treatments.

In this paper, concentration profiles away from grain boundaries in sensitized material have been calculated using thermodynamic and diffusion models and the results related to the degree of sensitization obtained from DL-EPR tests. In this way, it has been possible to access element concentration profiles right up to the $\mathrm{M}_{23} \mathrm{C}_{6}$ - austenite interface. As can be seen from Fig. 6, for example, the predicted profiles are very narrow, extending down to less than $5 \mathrm{~nm}$. The experimental verification of these would require extensive atom probe tomography measurements or STEM analysis and the depletion trends can be examined, which has been outside the scope of this work.

In the above analysis, for simplicity, any possible effect of the $\mathrm{C}, \mathrm{Ni}$ or $\mathrm{Si}$ concentration profiles on the electrochemical properties of the $\mathrm{Cr}$ depleted zones and thereby DOS values have been ignored. Their inclusion in a statistical analysis would only be possible with a much wider data base of alloy compositions and treatments. Their inclusion would be made simpler if a fundamental model for the effects of concentration gradients on measured DOS were available.

Further work is required to explore the validity of the above approach using other stainless steels with different chemical compositions. It is possible, for example, that the critical chromium concentration defining the width of the chromium depleted zone depends on the bulk alloy composition. Also, any possible effects of the Ni and Si concentration profiles at the grain boundaries on the measured DOS have been ignored. 


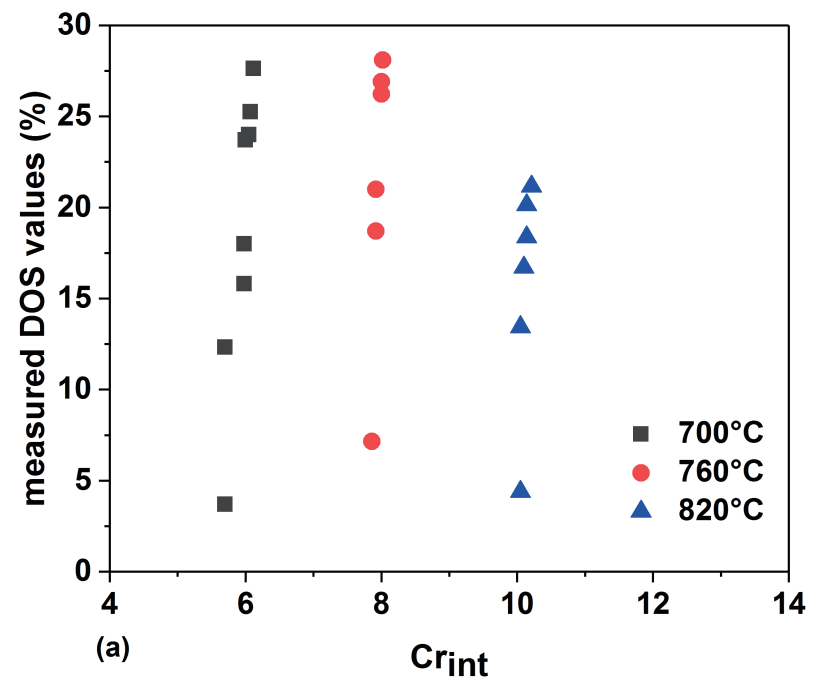

Fig. 11(a). Variation of measured DOS as a function of $\mathrm{Cr}_{\text {crit. }}$. (Online version in color.)

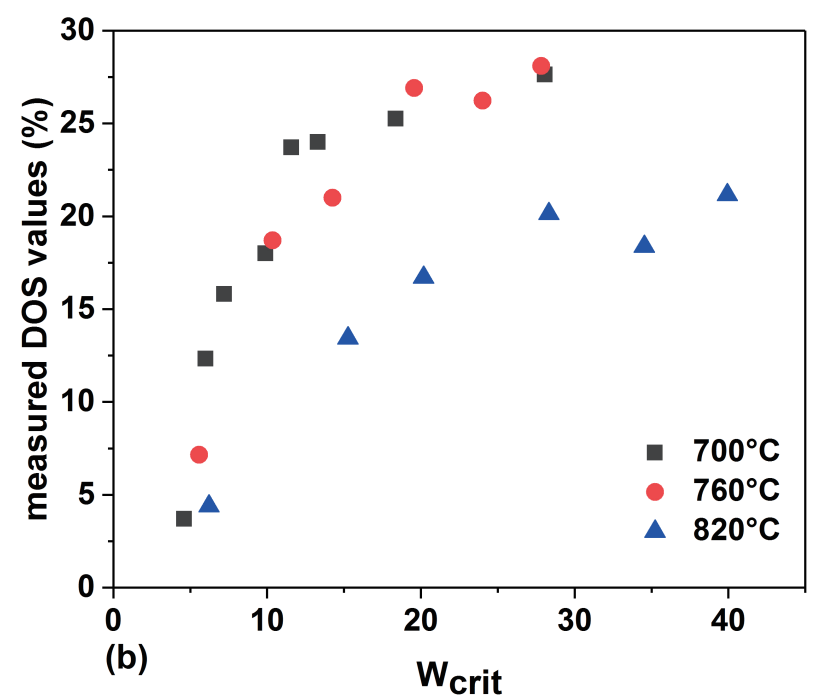

Fig. 11(b). Variation of measured DOS as a function of $\mathrm{W}_{\text {crit }}$. (Online version in color.)

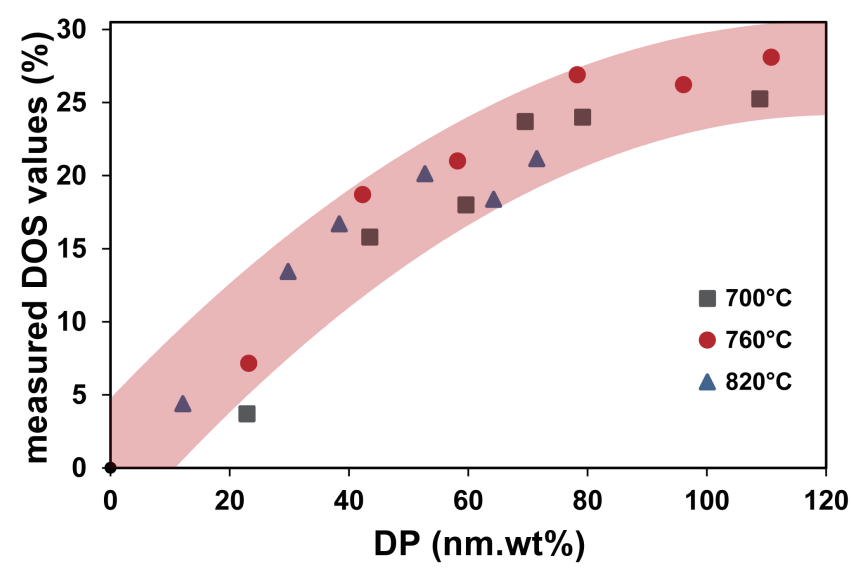

Fig. 11(c). Variation of measured DOS as a function of DP = $\left(12-\mathrm{Cr}_{\text {int }}\right) \cdot \mathrm{W}_{\text {crit }}$ (Online version in color.)

\section{Conclusions}

The degree of sensitization of an Fe-16.80Cr-6.36Ni$0.95 \mathrm{Si}-0.105 \mathrm{C}$ austenitic stainless steel subjected to isothermal heat treatments for times up to $80 \mathrm{mins}$ at 700 ,
760 and $820^{\circ} \mathrm{C}$ has been experimentally determined using DL-EPR testing and the results analysed in terms of the $\mathrm{Cr}$ concentration profiles obtained using thermodynamic and one-dimensional diffusion calculations. For the first time, such calculations have been made taking into account the multicomponent interaction effects of all the above alloying elements on the local $\mathrm{M}_{23} \mathrm{C}_{6}$ carbide - austenite matrix interface equilibrium, the atomic mobilities, and the flux balance at the interface. The experimental measurements show that sensitization, as measured by DOS vs time, is fastest at $760^{\circ} \mathrm{C}$ while it occurs at similar, slower rates at 700 and $820^{\circ} \mathrm{C}$. The thermodynamic and diffusion analyses show that DOS follows a non-linear correlation with a depletion parameter that depends on the width of the calculated $\mathrm{Cr}$ concentration profile at the critical $\mathrm{Cr}$ concentration of 12 wt. \% and the $\mathrm{Cr}$ concentration at the $\mathrm{M}_{23} \mathrm{C}_{6}$ - austenite interface calculated assuming local interface equilibrium and an interfacial flux balance for all alloying elements.

The approach adopted in the present work shows promise for implementation into a software tool for predicting precipitate growth, depletion profiles and sensitization as a function of composition and thermal history.

\section{Acknowledgements}

Funding from the European Union's Horizon 2020 research and innovation programme under the Marie Skłodowska-Curie grant agreement No 675715 is gratefully acknowledged.

\section{REFERENCES}

1) A. J. Sedriks: Corrosion of Stainless Steels, 2nd ed., John Wiley and Sons, New York, (1996), 112

2) ASTM G108-94: 2010, Standard Test Method for Electrochemical Reactivation (EPR) for detecting sensitization of AISI type 304 and 304L stainless steels.

3) SFS EN ISO 12732: 2008, Corrosion of metals and alloys Electrochemical potentiokinetic reactivation measurement using the double loop method (based on Cihal's method).

4) E. C. Bain, R. Aborn and J. Rutherford: Trans. Am. Soc. Steel Treat., 21 (1933), 481.

5) R. Lula, A. Lena and G. Kiefer: Trans. Am. Soc. Met., 46 (1954), 197.

6) R. Cowan and C. S. Tedmon: Intergranular Corrosion of Iron-NickelChromium Alloys, Vol. 3, Plenum Press, New York, (1973), 293.

7) E. L. Hall and C. L. Briant: Metall. Trans. A, 15 (1984), 793.

8) G. S. Was and V. B. Rajan: Corrosion, 43 (1987), 576.

9) W. E. Mayo: Mater. Sci. Eng. A, 232 (1997), 129

10) V. Kain, R. C. Prasad and P. K. De: Corrosion, 58 (2002), 15.

11) N. Parvathavarthini and U. K. Mudali: Corros. Rev., 32 (2014), 183.

12) S. M. Bruemmer: Corrosion, 46 (1990), 698.

13) C. Stawström and M. Hillert: J. Iron Steel Inst., 207 (1969), 77.

14) G. S. Was and R. M. Kruger: Acta Metall., 33 (1985), 841.

15) H. Sahlaoui, H. Sidhom and J. Philibert: Acta Mater., 50 (2002), 1383.

16) T. Sourmail and H. K. D. H. Bhadeshia: Calphad, 27 (2003), 169.

17) A. P. Majidi and M. A. Streicher: Corrosion, 40 (1984), 584

18) S. Rahimi, D. L. Engelberg and T. J. Marrow: Corros. Sci., 53 (2011), 4213.

19) M. Lewis and B. Hattersley: Acta Metall., 13 (1965), 1159

20) F. Beckitt and B. Clark: Acta Metall., 15 (1967), 113.

21) R. Hoffman and E. Turnbull: J. Appl. Phys., 22 (1951), 634

22) A. Atkinson: J. Phys. Colloq., 46 (1985), C4-379.

23) H. Aaron and H. I. Aaronson: Acta Metall., 16 (1968), 789.

24) S. M. Bruemmer: Corrosion, 46 (1990), 698

25) A. Borgenstam, L. Höglund, J. Ågren and A. Engström: J. Phase Equilib., 21 (2000), 269.

26) H. Larsson and R. C. Reed: Acta Mater, 56 (2008), 3754

27) T. Sourmail, C. H. Too and H. Bhadeshia: ISIJ Int., 43 (2003), 1814

28) Y. Yin, R. G. Faulkner, P. Moreton, I. Armson and P. Coyle: $J$. Mater. Sci., 45 (2010), 5872.

29) H. Sahlaoui, K. Makhlouf, H. Sidhom and J. Philibert: Mater. Sci. Eng. A, 372 (2004), 98. 